\title{
Pseudo-Rodlike Molecules with Hockey-Stick-Shaped Mesogen
}

E-Joon Choi, ${ }^{\text {a* }}$ Kyung-Min Park, ${ }^{\mathrm{a}}$ Dae-Yoon Kim, ${ }^{\mathrm{b}}$ Kwang-Un Jeong, ${ }^{\mathrm{b} *}$ and Ji-Hoon Lee ${ }^{\mathrm{c}}$

${ }^{a}$ Department of Polymer Science and Engineering, Kumoh National Institute of Technology, Gumi 730701, Korea.E-mail: ejchoi@kumoh.ac.kr.

${ }^{b}$ Department of Polymer-Nano Science and Technology, Chonbuk National University, Jeonju 561-756, Korea.E-mail: kujeong@jbnu.ac.kr.

${ }^{c}$ Advanced Electronics and Information Research Center, Division of Electronics Engineering, Chonbuk National University, Jeonju 561-756, Korea. 


\section{Supplementary Information}

\section{Experimental}

\subsection{Synthesis of compound $3 a$}

A mixture of compound 1 ( $0.6 \mathrm{~g}, 0.93 \mathrm{mmol})$, 2,3,4-trifluorobenzoic acid ( $0.16 \mathrm{~g}, 0.90 \mathrm{mmol}), \mathrm{DCC}(0.18$ $\mathrm{g}, 0.87 \mathrm{mmol})$, and DMAP $(0.01 \mathrm{~g}, 0.08 \mathrm{mmol})$ in DCM $(60 \mathrm{ml})$ was stirred at room temperature for $24 \mathrm{~h}$. The urea formed was removed by filtration, the filtrate was washed with $5 \%$ aqueous acetic acid and water, and the solvent was evaporated in vacuo. The solid residue was purified by flash column chromatography on silica gel using chloroform as eluent. Yield: 40\%; IR (KBr pellet, $\left.\mathrm{cm}^{-1}\right): 3075$ (aromatic C-H stretch), 2917, 2850 (aliphatic C-H stretch), 1739 (conj. C=O stretch), 1603, 1511 (aromatic $\mathrm{C}=\mathrm{C}$ stretch), 1273, 1193, $1166\left(\mathrm{C}-\mathrm{O}, \mathrm{C}-\mathrm{F}\right.$ stretch); ${ }^{1} \mathrm{H} \mathrm{NMR}\left(400 \mathrm{MHz}, \mathrm{CDCl}_{3}, \delta\right.$ in ppm): 8.35-8.25 (t, 4H, Ar- $\underline{\mathrm{H}}), 8.2-8.14(\mathrm{~d}, 1 \mathrm{H}, \mathrm{Ar}-\underline{\mathrm{H}}), 8.11-8.08$ (s, 1H, Ar- $\underline{\mathrm{H}}), 7.96-7.89$ (m, 1H, Ar- $\underline{\mathrm{H}})$,

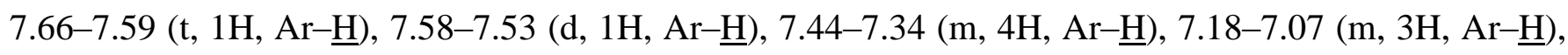
6.97-6.89 (d, 2H, Ar- $\underline{\mathrm{H}}$ ), 3.99-3.91 (t, 2H, Ar-O- $\underline{\mathrm{C}}_{2}-$ ), 1.83-1.72 (m, 2H, Ar-O- $\left.\mathrm{CH}_{2}-\underline{\mathrm{C}}_{2}-\right), 1.5-1.4$ (m, $\left.2 \mathrm{H}, \mathrm{Ar}-\mathrm{O}-\mathrm{CH}_{2}-\mathrm{CH}_{2}-\mathrm{C}_{2}-\right)$, 1.4-1.18 (m, 18H, $\left.-\underline{\mathrm{C}}_{2}-\right), 0.92-0.84$ (t, 3H, $\left.-\underline{\mathrm{CH}}_{3}\right)$; Anal. Calcd for $\mathrm{C}_{46} \mathrm{H}_{43} \mathrm{~F}_{3} \mathrm{O}_{9}$ : C 69.34, H 5.44; Found: C 69.93, H 5.75.

\subsection{Synthesis of compound $3 b$}

Quantities: compound 1 (0.5 g, $0.78 \mathrm{mmol})$, 3,4,5-trifluorobenzoic acid (0.13 g, $0.73 \mathrm{mmol})$, DCC (0.15 g, $0.72 \mathrm{mmol})$, and DMAP $(0.01 \mathrm{~g}, 0.08 \mathrm{mmol})$. The experimental procedure was as described for the preparation of compound 3a. Yield: 35\%; IR (KBr pellet, $\left.\mathrm{cm}^{-1}\right)$ : 3075 (aromatic C-H stretch), 2920, 2850 (aliphatic C-H stretch), 1736 (conj. C=O stretch), 1603, 1511 (aromatic C=C stretch), 1273, 1197, 1163 (C-O, C-F stretch); ${ }^{1} \mathrm{H}$ NMR (400 MHz, $\mathrm{CDCl}_{3}, \delta$ in ppm): 8.34-8.24 (t, 4H, Ar- $\underline{\mathrm{H}}$ ), 8.18-8.13 (d, 1H, $\operatorname{Ar}-\underline{\mathrm{H}}), 8.05-8.03(\mathrm{~s}, 1 \mathrm{H}, \mathrm{Ar}-\underline{\mathrm{H}}), 7.91-7.82(\mathrm{t}, 1 \mathrm{H}, \mathrm{Ar}-\underline{\mathrm{H}}), 7.65-7.58$ (t, 1H, Ar- $\underline{\mathrm{H}}), 7.54-7.48$ (d, 1H, $\operatorname{Ar}-\underline{\mathrm{H}}), 7.42-7.33$ (m, 4H, Ar- $\underline{\mathrm{H}}), 7.13-7.07$ (d, 2H, Ar- $\underline{\mathrm{H}}), 6.94-6.88$ (d, 2H, Ar- $\underline{\mathrm{H}}), 3.97-3.91$ (t, 2H, $\mathrm{Ar}-\mathrm{O}-\mathrm{C}_{2}-$ ), $1.81-1.72$ (m, 2H, Ar-O- $\left.\mathrm{CH}_{2}-\underline{\mathrm{C}}_{2}\right), 1.48-1.39$ (m, 2H, Ar-O- $\left.\mathrm{CH}_{2}-\mathrm{CH}_{2}-\underline{\mathrm{C}}_{2}\right), 1.37-1.16$ (m, 18H, $-\mathrm{C}_{2}-$ ), 0.9-0.83 (t, 3H, $-\underline{\mathrm{CH}}_{3}$ ); Anal. Calcd for $\mathrm{C}_{46} \mathrm{H}_{43} \mathrm{~F}_{3} \mathrm{O}_{9}$ : C 69.34, H 5.44; Found: C 69.93, H 5.75 .

\subsection{Synthesis of compound $3 c$}

Quantities: compound 1 (0.5 g, $0.78 \mathrm{mmol})$, 2,4,6-trifluorobenzoic acid (0.13 g, $0.73 \mathrm{mmol})$, DCC (0.15 g, $0.72 \mathrm{mmol})$, and DMAP (0.01 $\mathrm{g}, 0.08 \mathrm{mmol})$. The experimental procedure was as described for the preparation of compound 3a. Yield: 42\%; IR (KBr pellet, $\left.\mathrm{cm}^{-1}\right)$ : 3075 (aromatic C-H stretch), 2920, 2850 
(aliphatic C-H stretch), 1745 (conj. C=O stretch), 1603, 1511 (aromatic C=C stretch), 1263, 1197, 1166 (C-O, C-F stretch); ${ }^{1} \mathrm{H}$ NMR (400 MHz, $\mathrm{CDCl}_{3}, \delta$ in ppm): 8.32-8.24 (t, 4H, Ar- $\left.\underline{\mathrm{H}}\right), 8.17-8.12(\mathrm{~d}, 1 \mathrm{H}$, $\operatorname{Ar}-\underline{\mathrm{H}}), 8.09-8.07$ (s, 1H, Ar- $\underline{\mathrm{H}}), 7.64-7.57$ (t, 1H, Ar- $\underline{\mathrm{H}}), 7.57-7.52$ (d, 1H, Ar- $\underline{\mathrm{H}}), 7.44-7.33$ (m, 4H, $\operatorname{Ar}-\underline{\mathrm{H}}), 7.14-7.07(\mathrm{~d}, 2 \mathrm{H}, \mathrm{Ar}-\underline{\mathrm{H}}), 6.95-6.88(\mathrm{~d}, 2 \mathrm{H}, \mathrm{Ar}-\underline{\mathrm{H}}), 6.85-6.77$ (t, 2H, Ar- $\underline{\mathrm{H}}), 3.97-3.91$ (t, 2H, $\left.\mathrm{Ar}-\mathrm{O}-\mathrm{C}_{2}-\right)$, $1.81-1.72\left(\mathrm{~m}, 2 \mathrm{H}, \mathrm{Ar}-\mathrm{O}-\mathrm{CH}_{2}-\mathrm{C}_{2}-\right), 1.49-1.39$ (m, 2H, Ar-O- $\left.\mathrm{CH}_{2}-\mathrm{CH}_{2}-\mathrm{C}_{2}-\right), 1.38-$ 1.16 (m, 18H, $-\underline{\mathrm{CH}}_{2}-$ ), 0.9-0.83 (t, 3H, $-\underline{\mathrm{CH}}_{3}$ ); Anal. Calcd for $\mathrm{C}_{46} \mathrm{H}_{43} \mathrm{~F}_{3} \mathrm{O}_{9}$ : C 69.34, H 5.44; Found: $\mathrm{C}$ 69.68, H 5.79.
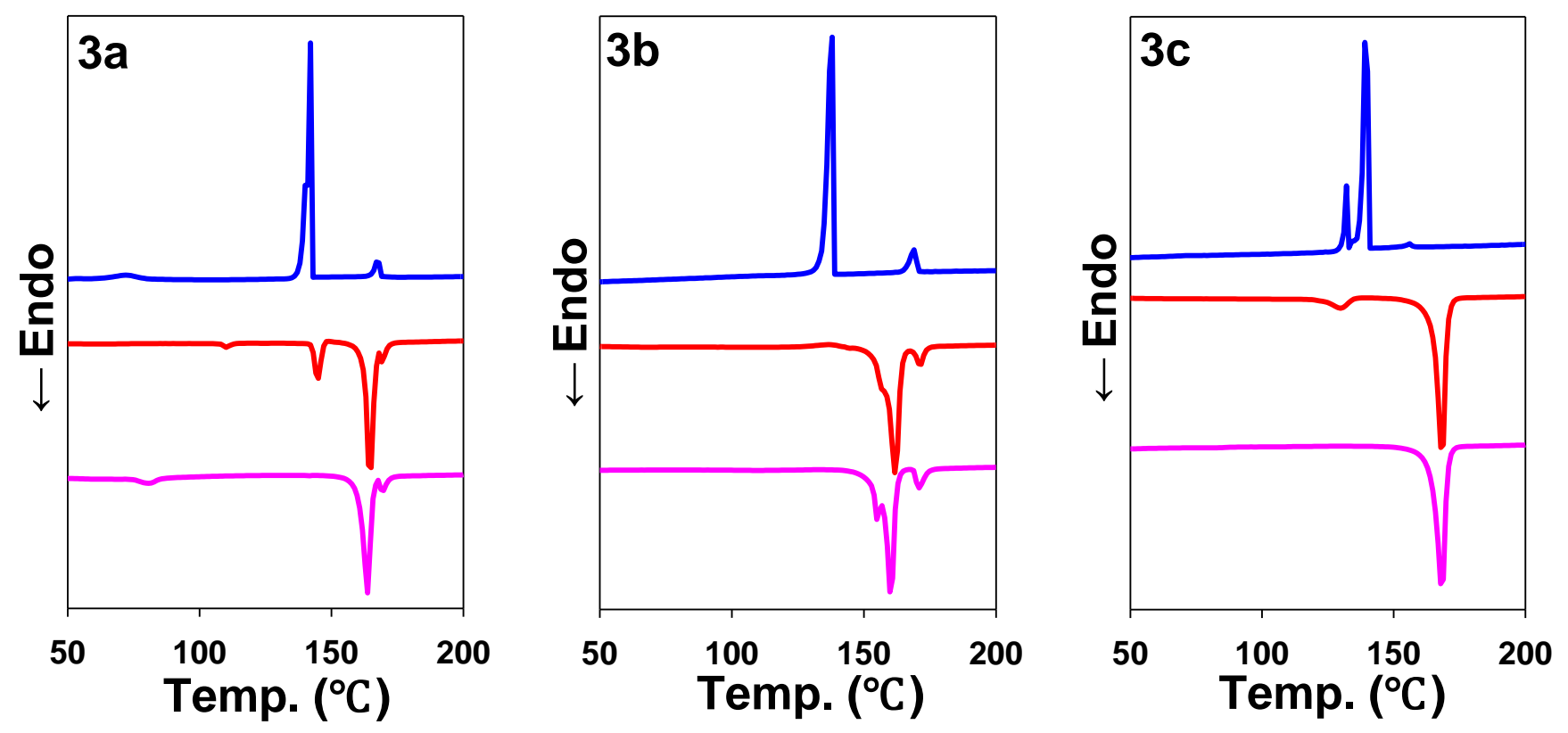

Figure 1. DSC thermograms of compounds (rate $=10{ }^{\circ} \mathrm{C} / \mathrm{min}$ ): lower curve: second heating; middle curve: first heating; upper curve: first cooling. 


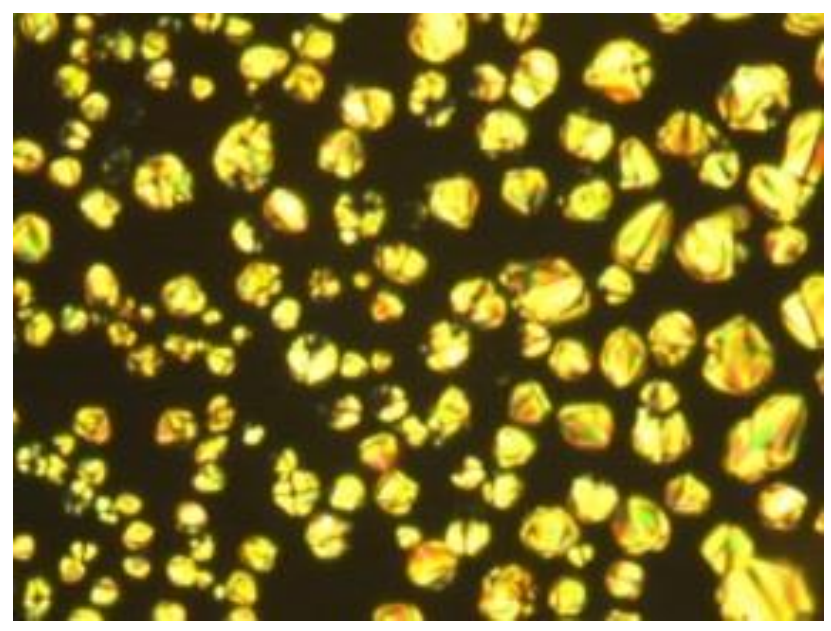

(a)

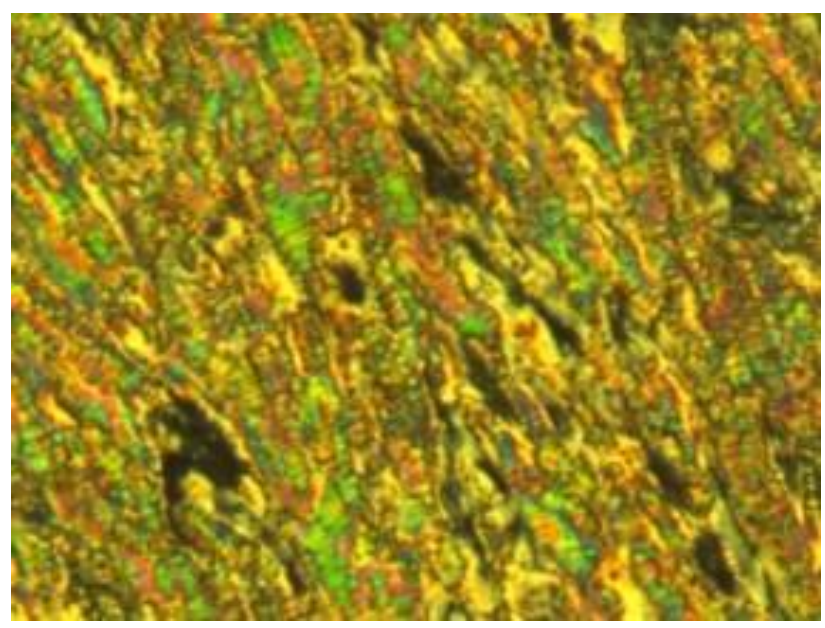

(c)

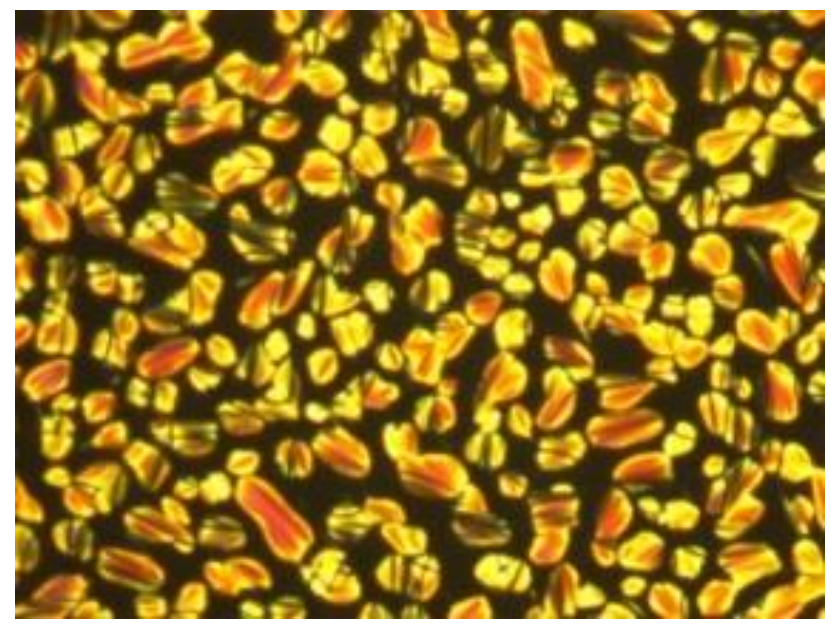

(e)

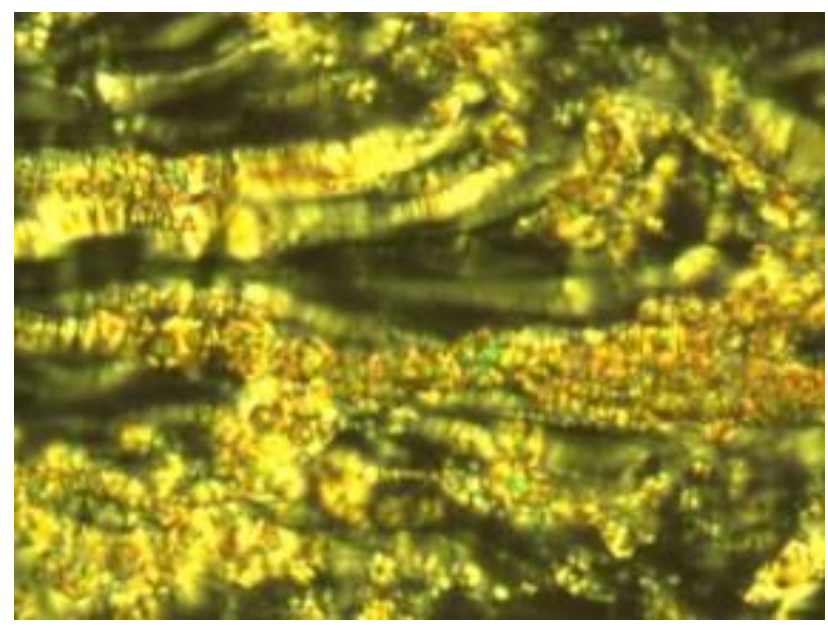

(b)

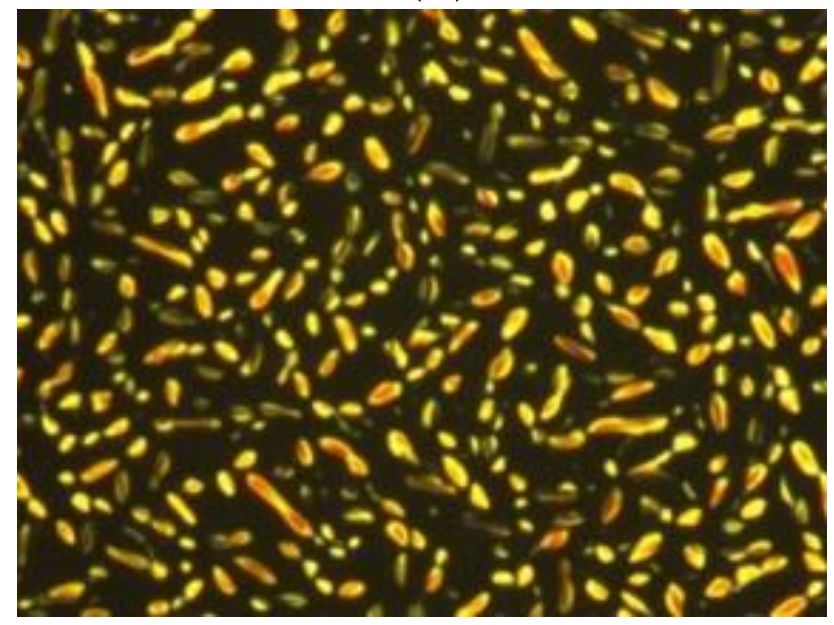

(d)

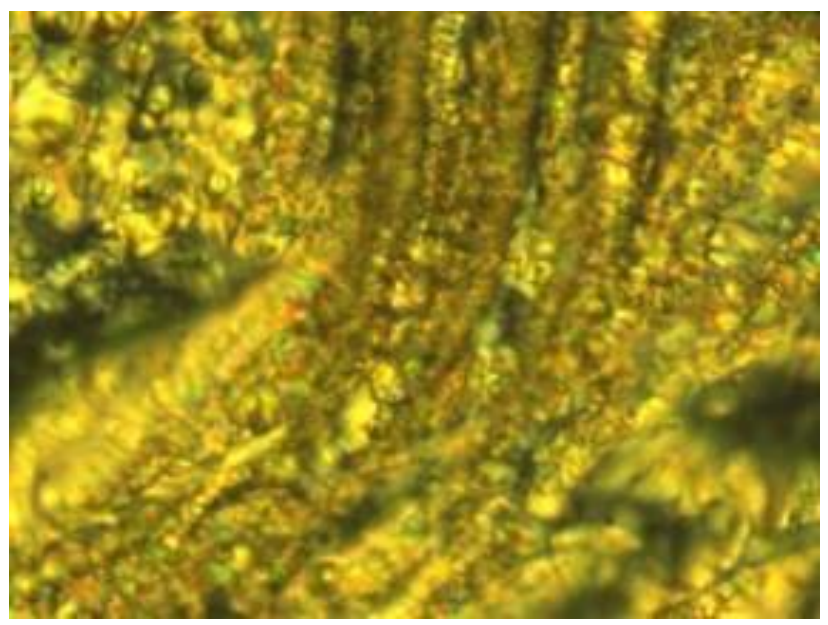

(f)

Figure 2. Cross-polarizing optical micrographs (magnification 200×). Compound 3a: (a) on cooling, $T=$ $168^{\circ} \mathrm{C}$; (b) on heating, $T=166^{\circ} \mathrm{C}$; (c) after shearing, $T=166^{\circ} \mathrm{C}$. Compound 3b: on cooling, (d) $T=$ $167^{\circ} \mathrm{C}$; (e) $T=163^{\circ} \mathrm{C}$; (f) on heating, $T=166^{\circ} \mathrm{C}$. 

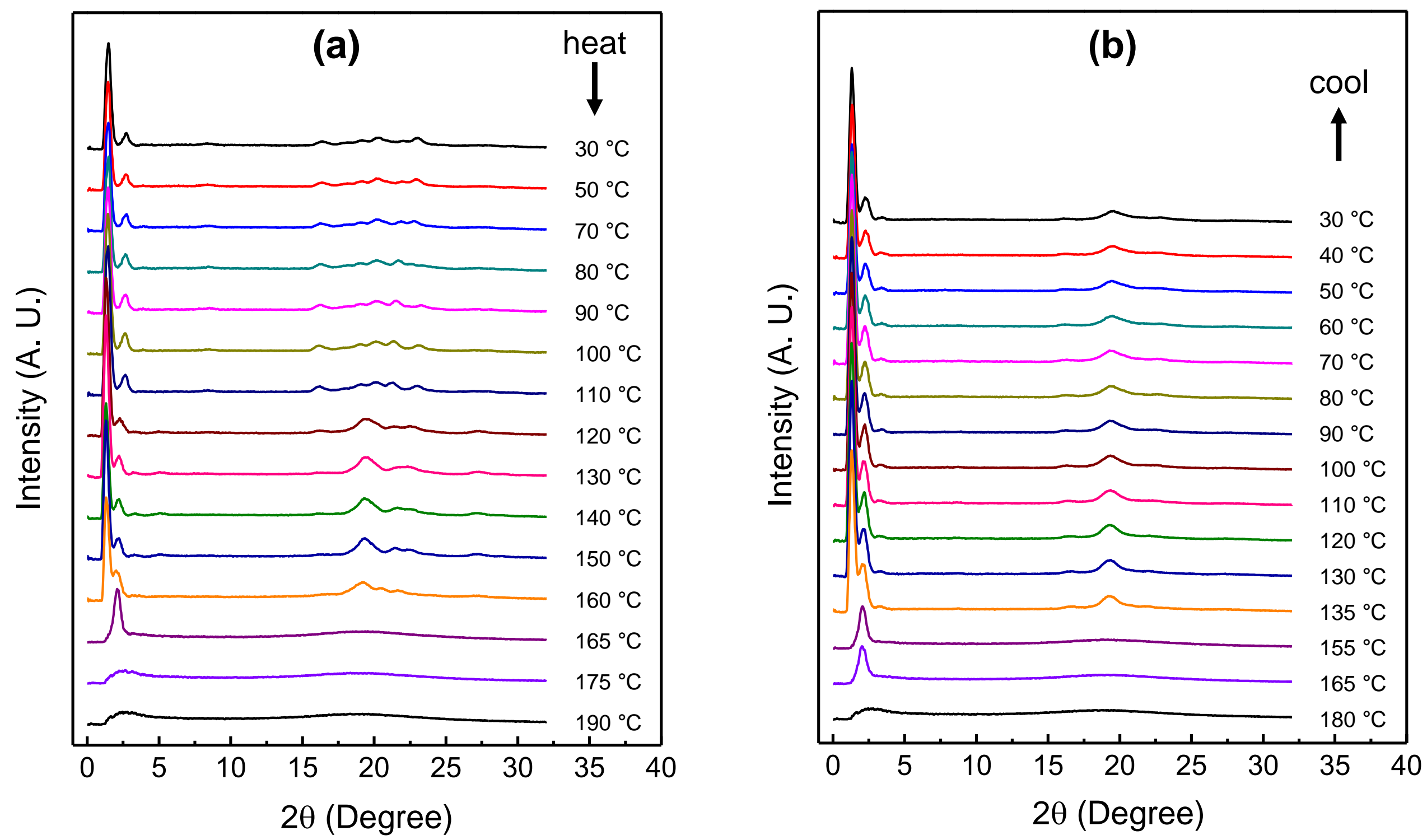

Figure 3. Sets of 1D WAXD powder patterns of $\mathbf{3 b}$ obtained at various temperatures during heating (a) and cooling (b) at a rate of $2.5^{\circ} \mathrm{C} / \mathrm{min}$. 